\title{
Evaluation of gold nanotracers to track adipose-derived stem cells in a PEGylated fibrin gel for dermal tissue engineering applications
}

This article was published in the following Dove Press journal:

International Journal of Nanomedicine

17 January 2013

Number of times this article has been viewed

\author{
Eunna Chung' \\ Seung Yun Nam ${ }^{1,2}$ \\ Laura M Ricles' \\ Stanislav $Y$ Emelianov ${ }^{1,2}$ \\ Laura J Suggs' \\ 'Department of Biomedical \\ Engineering, ${ }^{2}$ Department of Electrical \\ and Computer Engineering, \\ The University of Texas at Austin, \\ Austin, TX, USA
}

Correspondence: Laura J Suggs

Department of Biomedical Engineering,

The University of Texas

at Austin, I University

Station, C0800, Austin, TX 787I2, USA

$\mathrm{Tel}+|5| 2232$ |67|

Fax + I 512 47| 06I6

Email laura.suggs@mail.utexas.edu
Abstract: Evaluating the regenerative capacity of a tissue-engineered device in a noninvasive and synchronous manner is critical to determining the mechanisms for success in clinical applications. In particular, directly tracking implanted cells in a three-dimensional (3D) scaffold is desirable in that it enables the monitoring of cellular activity in a specific and localized manner. The authors' group has previously demonstrated that the PEGylation of fibrin results in a 3D scaffold that supports morphologic and phenotypic changes in mesenchymal stem cells that may be advantageous in wound healing applications. Recently, the authors have evaluated adipose-derived stem cells (ASCs) as a mesenchymal cell source to regenerate skin and blood vessels due to their potential for proliferation, differentiation, and production of growth factors. However, tracking and monitoring ASCs in a 3D scaffold, such as a PEGylated fibrin gel, have not yet been fully investigated. In the current paper, nanoscale gold spheres $(20 \mathrm{~nm})$ as cell tracers for ASCs cultured in a PEGylated fibrin gel were evaluated. An advanced dual-imaging modality combining ultrasound and photoacoustic imaging was utilized to monitor rat ASCs over time. The ASCs took up gold nanotracers and could be detected up to day 16 with high sensitivity using photoacoustic imaging. There were no detrimental effects on ASC morphology, network formation, proliferation, and protein expression/secretion (ie, smooth muscle $\alpha$-actin, vascular endothelial growth factor, matrix metalloproteinase-2, and matrix metalloproteinase-9) associated with gold nanotracers. Therefore, utilization of gold nanotracers can be an effective strategy to monitor the regenerative process of a stem cell source in a $3 \mathrm{D}$ gel for vascular and dermal tissue engineering applications.

Keywords: gold nanoparticles, adipose-derived stem cells, fibrin, ultrasound and photoacoustic imaging, angiogenesis, tissue engineering

\section{Introduction}

Tracking the regenerative process of cells that are seeded in a three-dimensional (3D) environment is essential for successful evaluation of a tissue-engineered system. This research goal can be achieved by applying biomedical imaging and nanomedicine techniques that have already been developed for clinical diagnosis and treatment. A variety of biomedical imaging strategies such as fluorescence microscopy, magnetic resonance imaging, and ultrasound (US) imaging, in particular for tissue engineering applications, have been investigated. ${ }^{1-3}$ These imaging modalities have been used to provide noninvasive, real-time evaluation of scaffold stability, ${ }^{4}$ biomacromolecule accumulation, ${ }^{5}$ and cellular activities. ${ }^{2,6,7}$ Among a number of biomedical imaging modalities, US imaging has been widely utilized in the clinic as well as the biomedical research field due to its simplicity and noninvasive operation. Photoacoustic (PA) 
imaging is a next-generation biomedical imaging technique that can be used in combination with US imaging. PA imaging can confer unique information due to the fact that the PA signal is specific to the optical absorption properties of contrast agents, such as gold nanoparticles, with high sensitivity, penetration depth, and potential for quantification. In particular, US or US/PA imaging has been used in tissue engineering to analyze protein production as well as cell tracking and scaffold structure..$^{5,6,8}$ Therefore, US and PA imaging in combination can map a cell/scaffold construct along with neighboring tissues and provide both morphological and functional information.

Nanoscale materials such as gold, iron oxides, and fluorochromes alone or with other carriers (eg, polymeric nanoparticles and liposomes) have been applied to detect or label cells and tissues of interest both in vitro and in vivo. ${ }^{6-11}$ For example, gold nanotracers (GNTs) have been proposed recently as a promising cell tracker, tagging and visualizing bone marrow-derived mesenchymal stem cells (BMSCs) easily in vitro without measurable negative influence on proliferation and differentiation towards cells of the mesenchyme. ${ }^{6}$ Furthermore, cells engulfing GNTs can be monitored and quantified effectively in vivo by means of a combined US/ PA imaging technique following injection into rat muscle. ${ }^{2}$ However, there is still a limited understanding of the longterm tracking and visualizing ability of gold nanoparticles for other cell types in a 3D hydrogel system for biomedical applications.

Adipose-derived stem cells (ASCs) have been evaluated as multipotent and clinically feasible, autologous, adult stem cell source that can differentiate into various cells such as adipocytes, chondrocytes, myocytes, and osteoblasts. ${ }^{12,13}$ Moreover, ASCs have been proposed as a promising cell source for vascular and skin engineering to regenerate vasculature and damaged skin due to their differentiation capacity into vascular cells and dermal cells as well as their potential to promote angiogenesis. ${ }^{14-16}$ For example, in the minipig burn injury model, treatment with adipose tissue showed improved wound closure and the regeneration of epidermis and blood vessels. ${ }^{17}$ ASCs have been highlighted as a superior stem cell source with respect to quantity and ease of isolation relative to BMSCs. ${ }^{13}$ ASCs are known to express a variety of surface markers (eg, CD29, CD90, and CD105), similar to BMSCs, and can be induced in vitro to express endothelial cell-like phenotypes and functionalities under the influence of chemical and physical cues. ${ }^{13,18}$ Furthermore, ASCs are known to have a paracrine effect by secreting growth fac- tors such as vascular endothelial growth factor (VEGF), transforming growth factor- $\beta$, and hepatocyte growth factor. ${ }^{19}$ Paracrine effects of ASCs on skin regeneration have been demonstrated by stimulating biological activities of keratinocytes and fibroblasts. ${ }^{20}$ However, the secretory profiles of ASCs in correlation with tissue regeneration are not yet fully understood.

Fibrin is known to be an effective culture platform for stem cells as well as endothelial cells in order to enhance angiogenesis. ${ }^{21,22}$ It is also known to induce ASC activity in vitro and in vivo. ${ }^{23,24} \mathrm{In}$ addition, it is commonly used in vascular tissue engineering due to its inherent angiogenic activity. For tissue regeneration, rapid angiogenesis is a critical step in maintaining a tissue-engineered scaffold. Fibrin-based gel scaffolds have also been developed to regenerate skin tissue. ${ }^{25}$ However, fibrin has certain characteristics such as a relatively fast degradation profile and limited mechanical properties that limit applications in dermal wound healing. The authors' group has investigated the chemical modification of fibrin for vascular and dermal tissue engineering applications. ${ }^{26-28}$ It was found that the PEGylation of fibrin gels can limit the loss of mechanical properties over time compared to unmodified fibrin. It was also demonstrated that BMSCs generate interconnected capillary-like networks in a PEGylated fibrin gel (PFG) after 1 week and show gene and protein expression characteristics of vascular differentiation both in vitro and in vivo.

The goal of the current study was to evaluate the feasibility of using GNTs as a stem cell tracker for vascular/ dermal tissue engineering using a 3D PFG system. A summary of the cell isolation, GNT labeling, and seeding in the PFG followed by US/PA imaging is shown in Figure 1. In order to validate the utility of this technique, the uptake of $20 \mathrm{~nm}$ GNTs by ASCs was investigated, longitudinal US/PA imaging of GNT-labeled ASCs in 3D PFGs over 16 days was performed, and the effects of GNTs on the biological and potential therapeutic properties of ASCs was evaluated.

\section{Material and methods Isolation and culture of ASCs}

Adipose tissue was harvested from the fat pads of Lewis rats (male, 9-12 weeks; Harlan Laboratories, Indianapolis, IN) and minced. Cells were isolated by digesting with $0.05 \%$ collagenase type I (Life Technologies, Carlsbad, CA) followed by repeated washing with Gibco ${ }^{\circledR}$ Hank's Balanced Salt Solution buffer supplemented with $1 \%$ penicillin-streptomycin 


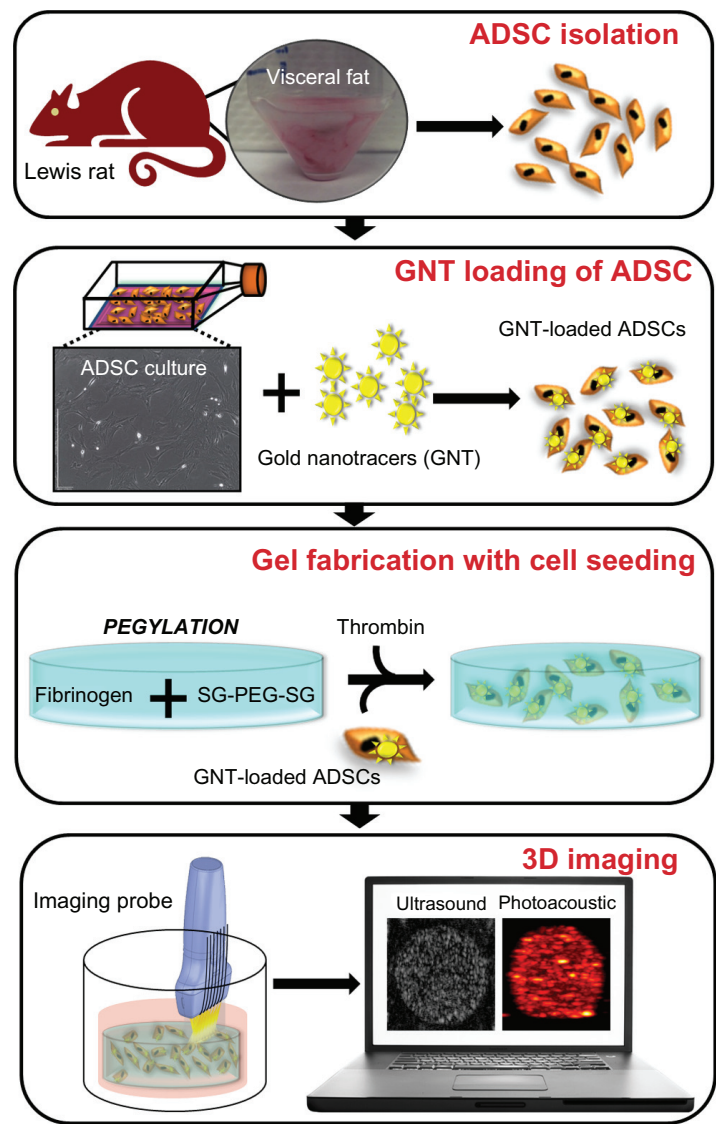

Figure I Schematic workflow for gold nanotracer-mediated tracking of adiposederived stem cells in a three-dimensional PEGylated fibrin gel.

Abbreviations: 3D, three-dimensional; ADSC, adipose-derived stem cell; GNT, gold nanotracer; SC-PEG-SC, succinimidyl glutarate polyethylene glycol.

(Life Technologies). Red blood cells were removed by centrifugation in a lysis buffer containing a 9:1 volume ratio of $160 \mathrm{mM}$ ammonium chloride and $17 \mathrm{mM}$ Tris base. Adherent cells were cultured in Dulbecco's modified Eagle's medium-low glucose with GlutaMAX ${ }^{\mathrm{TM}}$ I supplemented with $10 \%$ fetal bovine serum and $1 \%$ penicillin-streptomycin (Life Technologies). ASCs were passaged at a seeding density of $5000 \mathrm{cells} / \mathrm{cm}^{2}$. Passage two to five ASCs were used for all experiments in this study.

\section{Synthesis and treatment of GNTs}

GNTs $(20 \mathrm{~nm})$ were fabricated as described by Ricles et al. ${ }^{6}$ In brief, $1 \mathrm{~mL}$ of $10 \mathrm{mg} / \mathrm{mL}$ chloroauric acid (Sigma-Aldrich, St Louis, MO) was added into $97 \mathrm{~mL}$ purified water by a ultrapure water purification system (Barnstead Nanopure, ThermoFisher Scientific, Waltham, HA) and boiled on a hot plate at $400^{\circ} \mathrm{C}$. Then, $2 \mathrm{~mL}$ of $11.4 \mathrm{mg} / \mathrm{mL}$ sodium citrate (Sigma) was added with rapid stirring. GNTs were added to the cultured cells at a concentration of $4 \times 10^{7}$
GNTs/cell. The GNT-containing media was removed after 24 hours and replaced with the growth media for future cultivation.

\section{Preparation and culture of ASC-seeded PFGs}

For $1 \mathrm{~mL}$ PFG, $125 \mu \mathrm{L}$ fibrinogen $(80 \mathrm{mg} / \mathrm{mL}$; Sigma) solution in Dulbecco's phosphate buffered saline (DPBS; $\mathrm{pH}$ 7.8) was combined with $125 \mu \mathrm{L}$ succinimidyl glutarate polyethylene glycol solution $(8 \mathrm{mg} / \mathrm{ml}$; NOF America Corporation, White Plains, NY) and cells were seeded at 50,000 cells $/ \mathrm{mL}$ ( 250 micro-liter $\mu \mathrm{L}$ of $2 \times 10^{5}$ cells $\left./ \mathrm{ml}\right)$ followed by the addition of a thrombin solution (500 micro-liter $\mu \mathrm{L} 25 \mathrm{U}$, diluted with calcium chloride at $1: 3$ by volume; Sigma). After 10 minutes in a humidified carbon dioxide incubator at $37^{\circ} \mathrm{C}$, the culture media was added onto cell-seeded gels and replaced with fresh media after 30 minutes and again after 2 hours following gel fabrication. The culture medium was exchanged daily and cell growth was examined at days one, four, eight, and 16 using CellTiter $96^{\circledR}$ Aqueous One Solution Cell Proliferation Assay (containing methyl tetrazolium salt; Promega Corporation, Fitchburg, WI) according to the manufacturer's protocol. Afterwards, cells were fixed with $4 \%$ paraformaldehyde/DPBS for 45 minutes at each time point. The macroscopic image of each gel was acquired using a digital camera (DSC-W570, Sony) and the gel size was quantified with ImageJ software (1.45S) (National Institutes of Health, Bethesda, MD). Time points (days one, four, eight, and 16) in the current study denote that the assay was performed at a certain designated time after gel fabrication. For example, day one denotes that the assay was performed at 24 hours after cells were seeded in the gels.

\section{GNT analysis}

The absorbance spectra of GNT alone (in water and culture media) and GNT-loaded cells were taken from $300 \mathrm{~nm}$ to $999 \mathrm{~nm}$ at $2 \mathrm{~nm}$ intervals using a microplate reader (Synergy $^{\text {TM }}$ HT; BioTek Instruments, Winooski, VT). The data was normalized relative to the maximum absorbance. Furthermore, GNTs were loaded into cultured ASCs as described above. After 24 hours of incubation with the GNT-containing culture media, the cell pellets were prepared. As a negative control, cells without GNT treatment were also prepared in an identical manner. Cell pellets were fixed using (1) glutaraldehyde/paraformaldehyde and (2) osmium tetroxide/potassium ferrocyanide fixatives. Cell pellets were entrapped in an agar solution and 


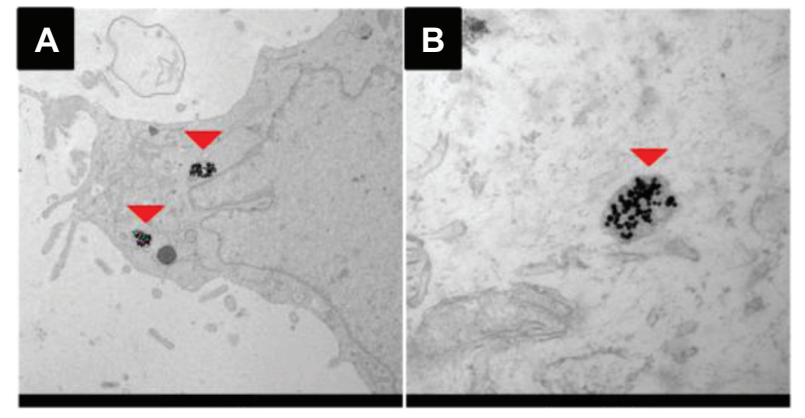

C

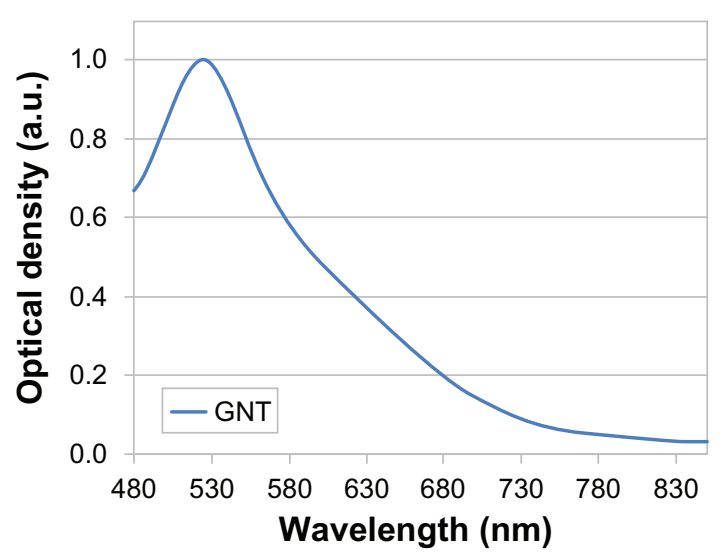

D

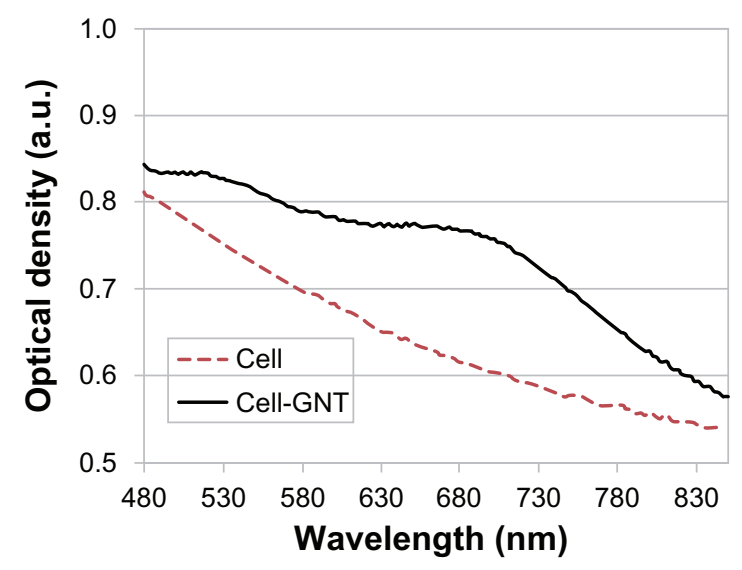

Figure 2 (A and $\mathbf{B}$ ) Transmission electron microscope images of gold nanotracerloaded adipose-derived stem cells. Red arrows indicate aggregated $20 \mathrm{~nm}$ gold nanotracers in the cytoplasm. (C) Ultraviolet-visible spectrum of gold nanotracers. (D) Ultraviolet-visible spectrum of cells and gold nanotracer-loaded cells. Abbreviations: GNT, gold nanotracer; a.u., arbitrary unit.

dehydrated using serial concentrations of ethanol solutions at a range of $50 \%-100 \%$, followed by $100 \%$ acetone treatment. The cell/agar pellets were incubated in a sequential series $(30 \%, 66 \%$, and $100 \%)$ of resin/acetone solutions and solidified in fresh $100 \%$ resin for a minimum of 2 days. Using a microwave device (Blowave, Pelco), the sample preparation for transmission electron microscopy analysis was accelerated. After resin molding, the samples were cut into nanoscale $(\sim 70 \mathrm{~nm})$ sections using an Ultracut UTC Ultramicrotome (Leica Microsystems, Wetzlar, Germany) and mounted onto a thin bar mesh grid made of copper for imaging.

\section{Bright field/phase contrast/dark field/ fluorescence imaging of silver-enhanced/ 4' 6-diamidino-2-phenylindole (DAPI)-stained cells}

For evaluation of GNT tracking, GNT-loaded ASCs were fixed and then permeabilized using $0.25 \%$ Triton $^{\mathrm{TM}}$ X-100 (Sigma)/DPBS. To increase the signal from GNTs, a silver enhancement method was applied by treating with a LI Silver ${ }^{\mathrm{TM}}$ Enhancement Kit (Nanoprobes, Inc, Yaphank, NY) for 30 minutes. ASCs were visualized using a bright field/phase contrast/dark field/fluorescence microscope (DMI3000 B; Leica) with overlay function following nuclear counterstaining using a VECTASHIELD Mounting Medium with DAPI (Vector Laboratories, Burlingame, CA).

\section{US/PA imaging}

Gels were placed on a tissue-mimicking phantom made of polyvinyl alcohol to reduce reverberation artifact and were then submerged in DPBS. To generate PA signals at a wavelength of $750 \mathrm{~nm}$, the gels were irradiated by a pulsed laser beam using a tunable optical parametric oscillating laser system (GWU, Inc, Erftstadt, Germany) and pumped by Nd:YAG laser (Spectra Physics, Inc, Mountain View, CA). Both US and PA signals were obtained using a Vevo ${ }^{\circledR} 2100$ US imaging system (VisualSonics, Inc, Toronto, Canada) and an imaging probe composed of a $20 \mathrm{MHz}$ US array transducer (VisualSonics) and a custom-made optical fiber bundle. After acquiring signals from the gels, post processing was performed to compensate the laser energy fluctuation, reduce noise, and quantify the signal intensities. The processed signals were interpolated to generate 3D US and PA images and 3D gel morphology images. The top-view PA images were created by maximum intensity projection in the axial direction. The volume of the gel was calculated based on the number of voxels from the region of the gel and the voxel size of the 3D gel morphology image. Total PA signal intensity was quantified by summing PA signals from each gel at a wavelength of $750 \mathrm{~nm}$, and the PA signal intensity per volume was calculated by dividing the total PA signal intensity by the calculated volume of the gel. 
VEGF, matrix metalloproteinase-2 (MMP-2), and matrix metalloproteinase-9 (MMP-9) enzyme-linked immunosorbent assay analyses

The quantification of secreted protein into the culture media was analyzed using commercially available enzyme-linked immunosorbent assay kits (R\&D Systems, Minneapolis, $\mathrm{MN}$ ) according to the manufacturer's protocol. The supernatant in the cell/gel system after each 24-hour period was collected.

\section{Smooth muscle $\alpha$-actin (SMA) immunofluorescence staining}

Gel samples were cryosectioned (12 micron thickness) following fixation using 4\% paraformaldehyde/DPBS solution for 45 minutes and cryoprotected using a gradient series of sucrose solutions $(5 \%-20 \%)$. These cryosectioned slides were used for all histological and immunofluorescence staining. For SMA, 0.1\% Triton X-100/DPBS was used for permeabilization. Immediately prior to incubation in the diluted primary antibody solution using mouse anti-SMA antibody (Abcam, Cambridge, United Kingdom) in 1\% normal serum overnight at $4^{\circ} \mathrm{C}, 10 \%$ goat normal serum/DPBS was used as a blocking buffer for 1 hour. As a negative control, normal immunoglobulin $G$ was used instead of the primary antibody solution. Sections were then washed using DPBS and were incubated in the diluted secondary antibody solution of Alexa Fluor ${ }^{\circledR} 488$ Goat Anti-Mouse Immunoglobulin $\mathrm{G}$ (Life Technologies) for 1 hour. The fluorescence signal was acquired using a fluorescence microscope (DMI3000 B; Leica) followed by mounting with a DAPI-including mounting media (Vector Laboratories).

\section{Statistical analysis}

All quantitative data were represented as mean \pm standard deviation. Repeated independent samples were three or four per test groups except the spectrum analysis. Statistical significance was analyzed by one-way or two-way analysis of variance with Tukey's multiple mean comparison test $(P<0.05)$.

\section{Results \\ GNT uptake of ASCs}

After 24-hour incubation with $20 \mathrm{~nm}$ GNTs, the ASC monolayer was investigated for uptake. Transmission electron microscope images show GNT aggregation in endocytotic

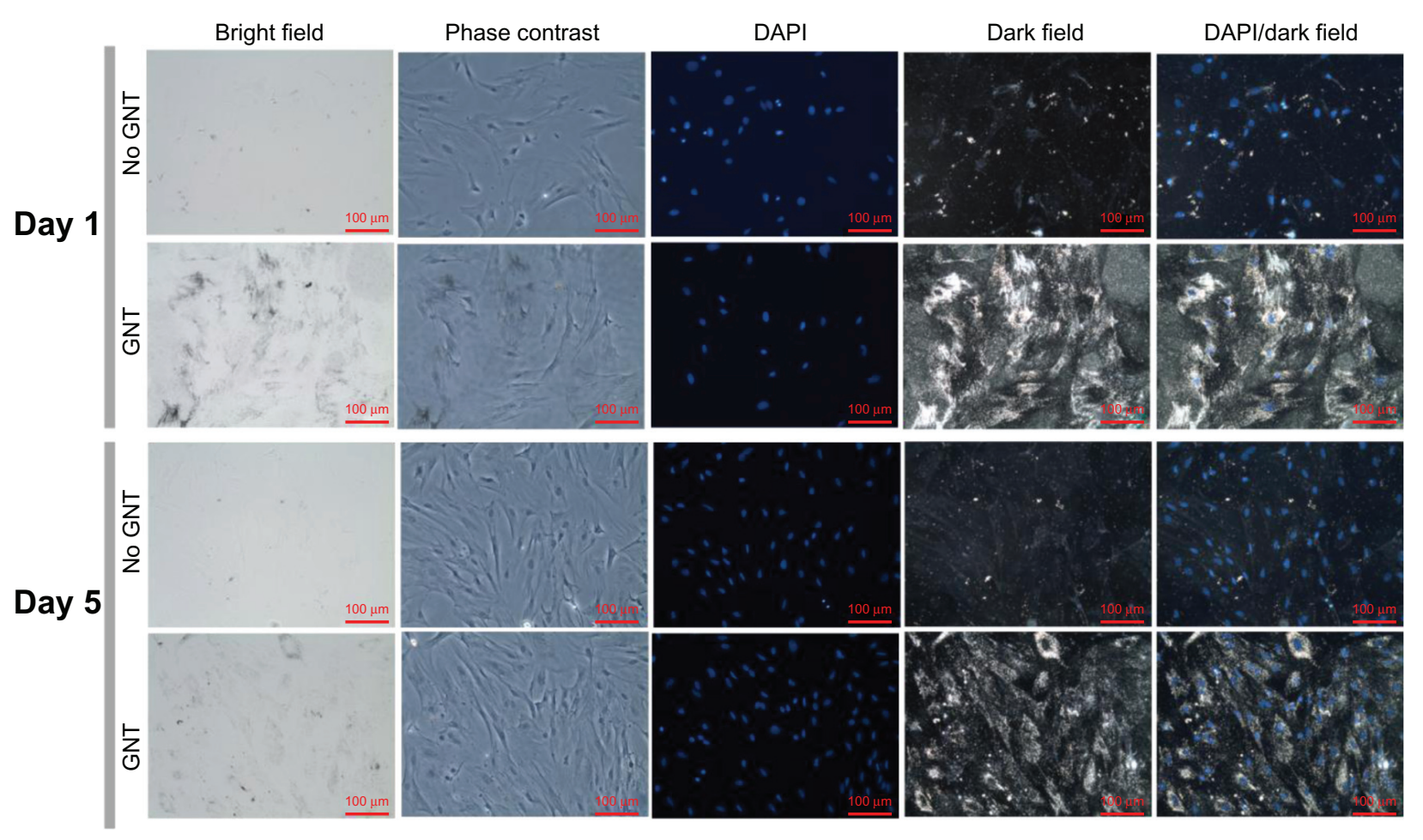

Figure 3 Bright field, phase contrast, and dark field optical images of monolayer adipose-derived stem cells with and without gold nanotracers on day I and day 5 . Abbreviations: DAPI, 4',6-diamidino-2-phenylindole; GNT, gold nanotracer. 


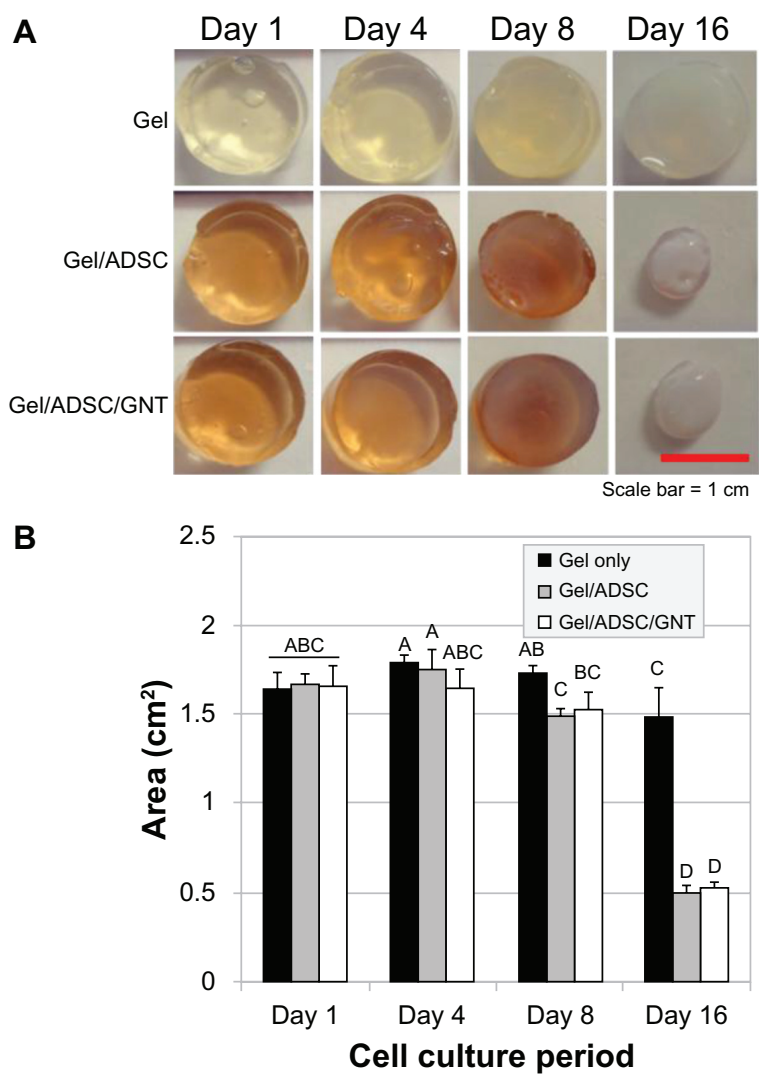

C

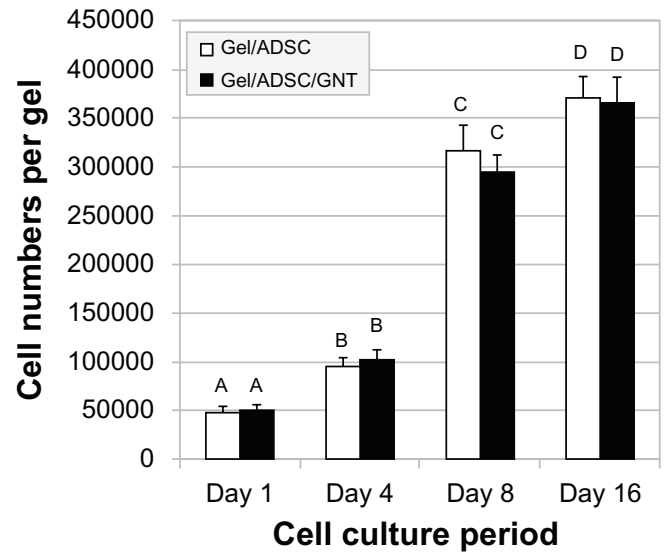

Figure $4 \mathrm{Gel}$ degradation of a PEGylated fibrin gel over time. (A) Digital camera photos of gels on days one, four, eight, and I6. (B) Quantification of the surface area of the gels from the front view. (C) Proliferation of gold nanotracer-loaded adiposederived stem cells in the PEGylated fibrin gel.

Notes: A methyl tetrazolium salt assay was used to derive cell numbers in adipose-derived stem cells with and without gold nanotracers in the gel over time. The data levels that do not share the same letters are significantly different $(P<0.05)$.

Abbreviations: ADSC, adipose-derived stem cell; GNT, gold nanotracer.

vesicles, located within the cytoplasm (Figure $2 \mathrm{~A}$ and $\mathrm{B}$ ). At $\sim 525 \mathrm{~nm}$, GNTs demonstrated a maximum optical density in both water and media. UV-visible spectra demonstrated that the loading of ASCs with GNTs conferred an increase in optical density at wavelengths of $\sim 600-750 \mathrm{~nm}$ compared to
ASCs without GNT treatment. In addition, GNT-loaded ASCs were detected in black and orange-yellow in the bright field and dark field images, respectively, in spite of a background signal due to silver enhancement (Figure 3). The majority of the GNT signal was localized in the cytoplasmic zone compared to the DAPI-stained nuclei. The GNT signal at both day 1 and 5 were greater compared to the corresponding no GNT conditions.

\section{D culture of ASC using PFG}

ASCs were cultured in PFGs for up to 16 days in this study. Cell proliferation and gel degradation were observed at four separate time points (days one, four, eight, and 16) (Figure 4). ASCs, whether loaded with GNTs or not, proliferated continuously for up to 16 days. From days four to eight, proliferation dramatically increased (approximately three-fold per gel). All PFGs (ie, gel alone, gel with ASCs, and gel with ASCs/GNT) maintained their general shape and size up to day eight. However, in contrast to the gel without cells, the size of the gels with ASCs or ASCs/GNT dramatically decreased. The gels turned whitish and opaque by the second week, indicative of significant cell-mediated degradation (days eight to 16).

\section{US and PA imaging of ASC/GNT}

Combining two imaging techniques, ie, US and PA imaging, allowed for the visualization of both overall gel geometry as well as the specific presence of GNT-loaded ASCs over time (Figure 5A-D). As shown in Figure 5A, the 3D gel image clearly shows the shape and size of the gel at each time point. The volume of all gels was quantified and is presented in Figure 5C. Both 3D images and quantitative analysis indicate significant volume contraction of the gel on days 16. The US images gave gel shape information and acoustic properties of the gels. As shown in the PA images in Figure 5A, the GNT-loaded ASCs generated strong PA signals at a wavelength of $750 \mathrm{~nm}$ due to the optical absorption peak broadening of GNTs. The PA signal intensities from all gels were quantified and are presented in Figure 5B and D. The total PA signal intensity over the entire gel volume (Figure 5D) is proportional to the total number of GNTs, which did not show any significant differences between time points. On the other hand, the PA signal intensity per volume (Figure 5B) relies on the GNT concentration, and it was remarkably increased at day 16 mainly due to volume contraction, which was also demonstrated in the PA image at day 16 with high contrast. 


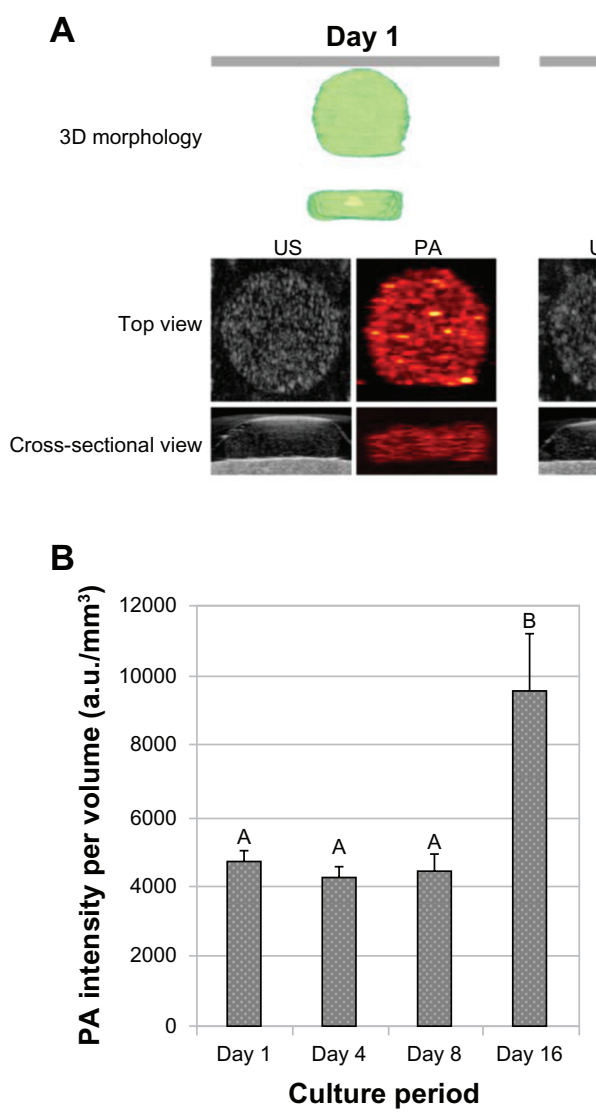

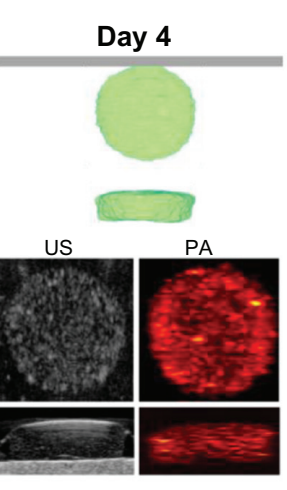

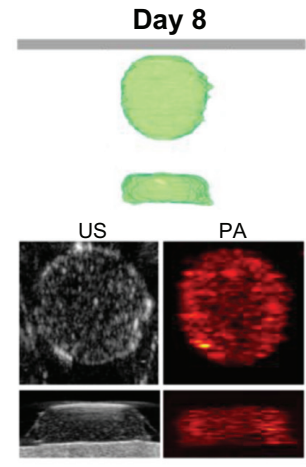

C

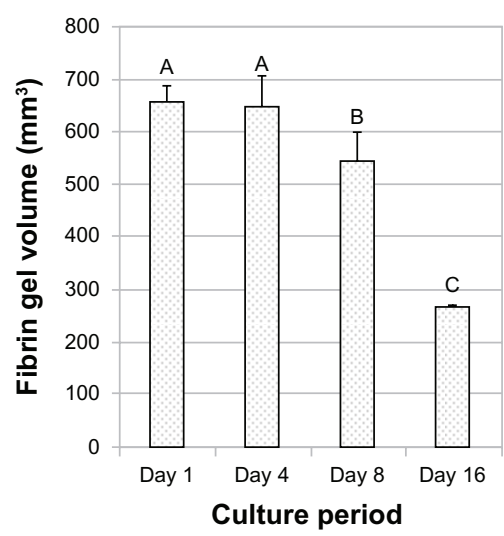

Day 16

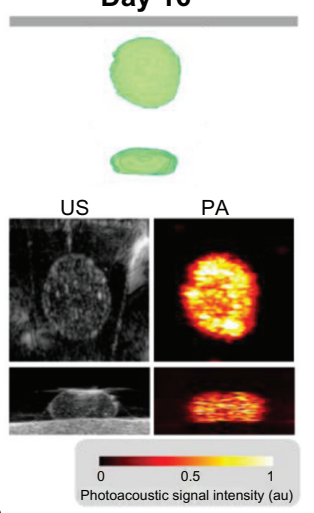

D

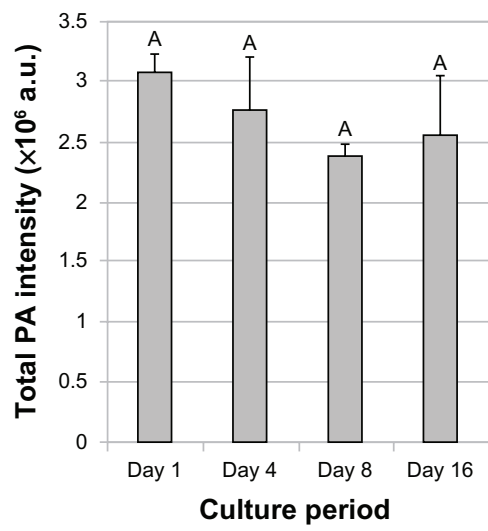

Figure 5 Dual ultrasound and photoacoustic imaging of adipose-derived stem cell-cultured PEGylated fibrin gels. (A) Reconstructed morphology of the fibrin gels (top two) and ultrasound/photoacoustic images at the center cross-section of the gels (bottom two). (B) Photoacoustic signal intensity per volume corresponding to the photoacoustic images in Figure 5A. (C) Fibrin gel volume. (D) Total photoacoustic signal intensity.

Note: The data levels that do not share the same letters are significantly different $(P<0.05)$.

Abbreviations: 3D, three-dimensional; PA, photoacoustic; US, ultrasound; a.u., arbitrary unit.

\section{Paracrine effects of ASCs in a 3D system: VEGF, MMP-2, and MMP-9}

Three angiogenesis-related proteins - VEGF, MMP-2, and MMP-9 - were chosen in the current study. Both total concentration and normalized amounts per cell of each protein are shown in Figures 6-8. Total secreted VEGF amounts increased over time. VEGF amounts were normalized to cell number, derived from the methyl tetrazolium salt proliferation assay. The normalized VEGF amount per cell, in contrast to the total amount, did not increase significantly over time. Likewise, the total amount of secreted MMP-2 increased over time but the amount per cell was maintained over the time course. Unlike MMP-2, the total amount of MMP-9 showed a maximum secretion on day eight and dramatically decreased to day 16 . The highest level of normalized MMP-9 was on day four.

\section{Immunofluorescence expression of SMA}

There was no significant difference in SMA expression between GNT-treated and control groups at all time points (Figure 9).
Initially (day one), ASCs showed strong SMA expression and the cells were spindle shaped and not interconnected. On day eight, SMA-positive ASCs with connected, tube-like morphogenesis were observed. Overall, the SMA signal decreased dramatically to day 16 . However, the level of SMA immunofluorescence was variable between cells.

\section{Discussion}

In tissue engineering and cell therapy, successful cell tracking can be approached using biocompatible contrast agents. Nanosize gold particles were chosen as an ASC tracer to apply next-generation biomedical imaging modalities to skin tissue engineering. In the current study, it was demonstrated that (1) ASCs can endocytose gold nanoparticles successfully, (2) these gold nanoparticles can be used to track ASCs in a 3D gel noninvasively using a combined US and PA imaging technique, and (3) this strategy does not affect cellular activities important in wound healing such as proliferation and phenotypic protein production by ASCs. Therefore, this 

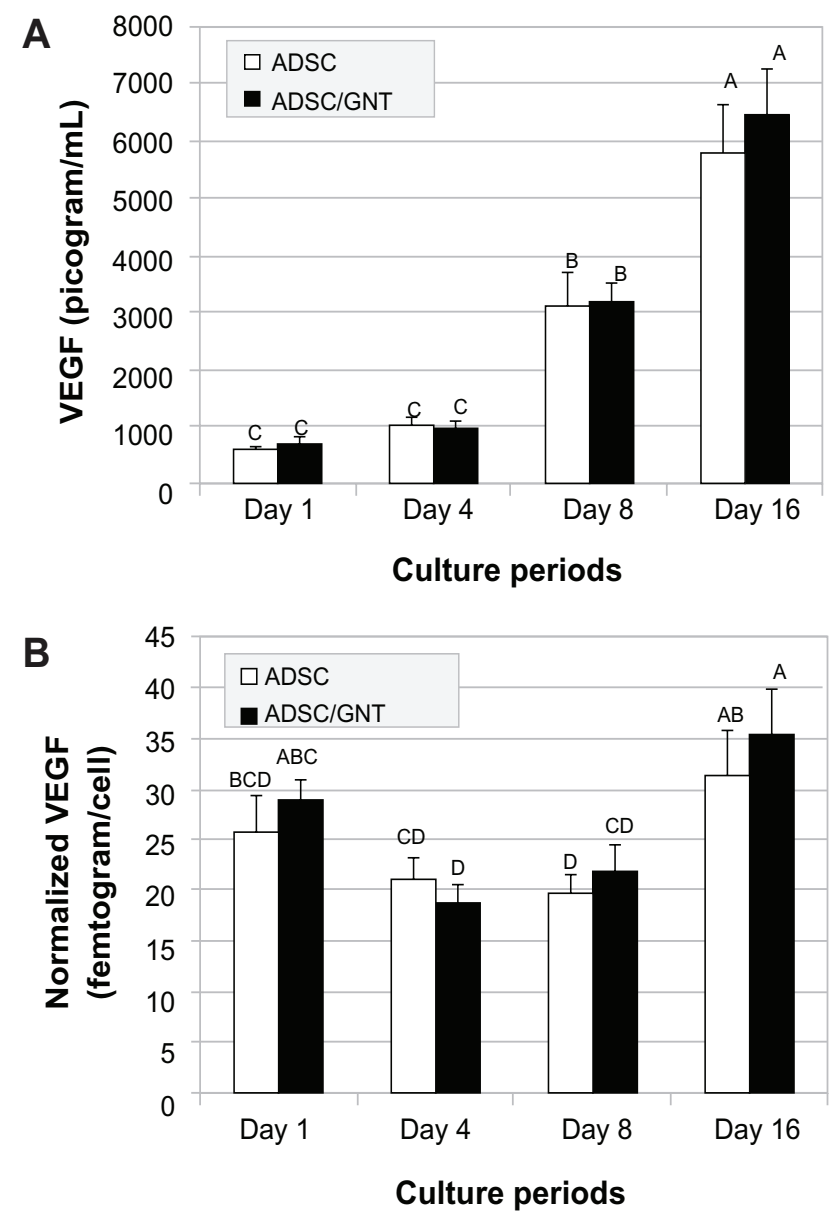

Figure 6 Vascular endothelial growth factor secretion from adipose-derived stem cells in a PEGylated gel. (A) Total amount of vascular endothelial growth factor secretion in an individual culture system. (B) Normalized amount of vascular endothelial growth factor secretion per cell.

Note: The data levels that do not share the same letters are significantly different $(P<0.05)$.

Abbreviations: ADSC, adipose-derived stem cell; GNT, gold nanotracer; VEGF, vascular endothelial growth factor.

study strongly suggests the feasibility of GNTs as a cell tracer for tissue applications.

According to the authors' previous work, BMSCs cultured in PFGs showed superior tubulogenesis in vitro compared to unmodified fibrin gels. In addition, PFG demonstrated greater mechanical stability than fibrin alone. In the current study, ASC-seeded gels showed accelerated degradation of PFG relative to controls (without cells). It is presumed that ASCs in the gel express proteases in order to facilitate matrix degradation. There were no differences, however, in matrix degradation by ASCs loaded with GNTs. Similar to BMSC tubulogenesis, ASCs rapidly formed interconnected networks within several days in PFG. Proliferation, however, increased throughout the culture period and was maintained even though significant gel degradation was observed on day 16.
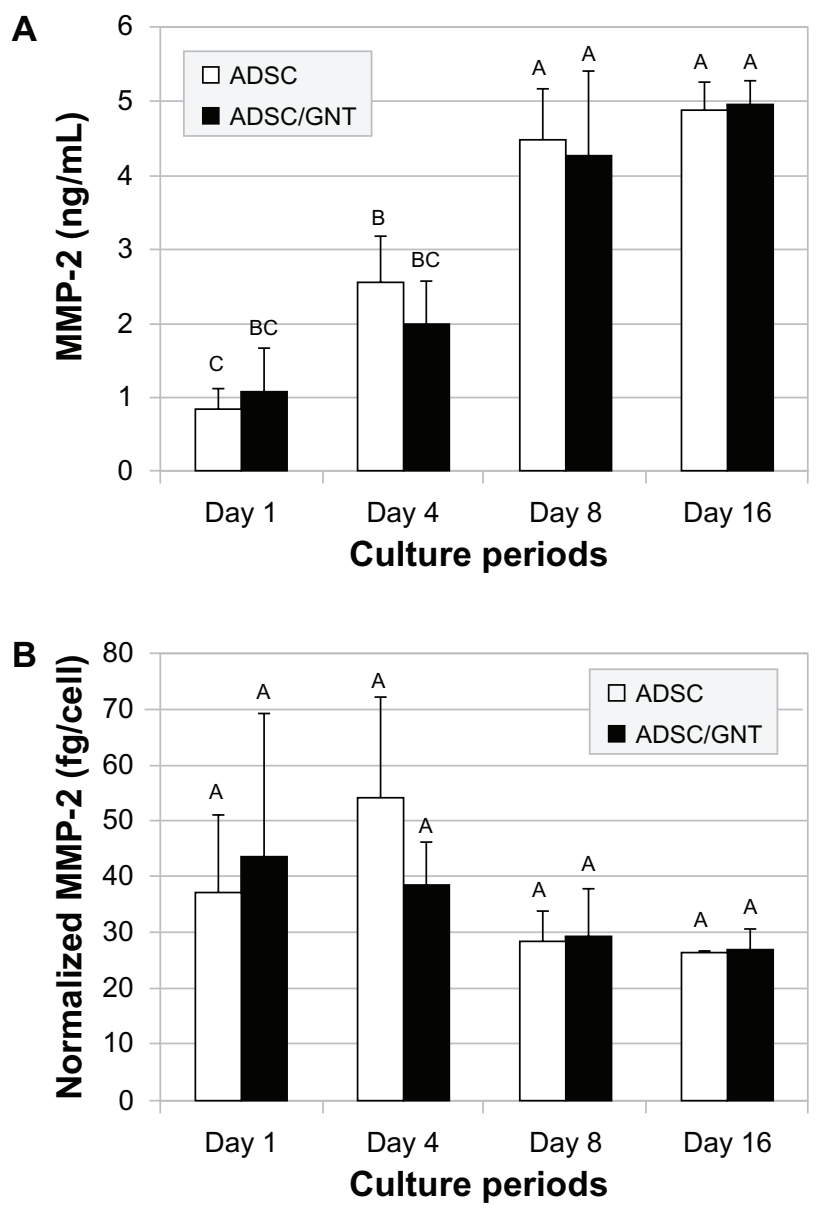

Figure 7 Matrix metalloproteinase-2 secretion from adipose-derived stem cells in a PEGylated gel. (A) Total amount of matrix metalloproteinase-2 secretion in a individual culture system. (B) Normalized amount of matrix metalloproteinase-2 secretion per cell.

Note: The data levels that do not share the same letters are significantly different $(P<0.05)$.

Abbreviations: ADSC, adipose-derived stem cell; GNT, gold nanotracer; MMP-2, matrix metalloproteinase- 2 .

Techniques to evaluate tissue regeneration, such as histologic or immunohistochemical staining, require additional time- and cost-consuming procedures following tissue sectioning. Various techniques and materials have been applied to label and track ASCs in situ in both in vitro and in vivo studies. Lequeux et al employed bromodeoxyuridine nuclear labeling to track ASCs. ${ }^{7}$ In addition, Wolbank et al utilized reporter genes of luciferase and green fluorescent protein for tracking in vitro differentiation and in vivo localization of ASCs. ${ }^{29}$ These techniques trace stem cells for long time periods, but real-time 3D reconstructed imaging/ mapping with cell tracking is not feasible.

The current work demonstrated the long-term tracking of ASCs in PFG using GNTs and a dual US and PA imaging system, as the material changed over time. In the case of rat ASCs, aggregated GNTs in the endocytic vesicles were 
A
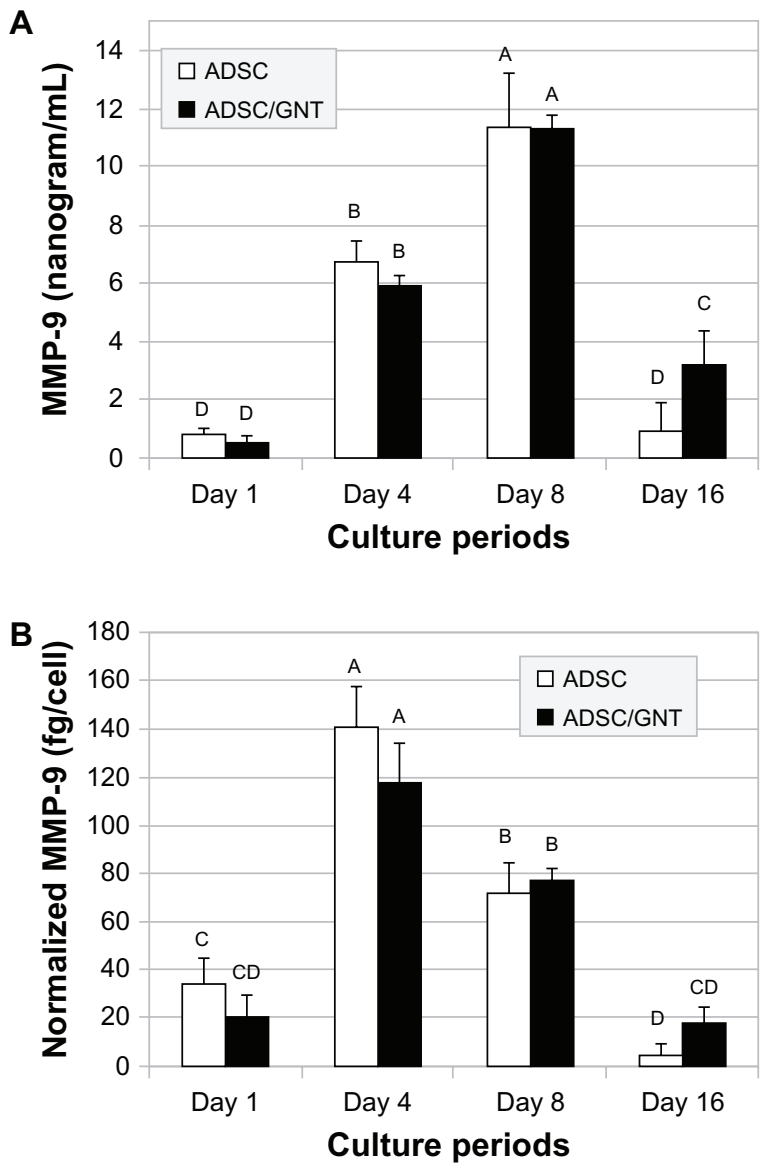

Figure 8 Matrix metalloproteinase- 9 secretion from adipose-derived stem cells in a PEGylated gel. (A) Total amount of matrix metalloproteinase-9 secretion in an individual culture system. (B) Normalized amount of matrix metalloproteinase- 9 secretion per cell.

Note: The data levels that do not share the same letters are significantly different $(P<0.05)$.

Abbreviations: ADSC, adipose-derived stem cell; GNT, gold nanotracer; MMP-9, matrix metalloproteinase-9.

observed at 24 hours after treatment. It is known that both somatic and stem cells take up nanoparticles via early endosomes, are stored in late endosomes and lysosomes, and are eventually excreted from the cell. ${ }^{31}$ In the current study, strong PA signals were detected from the ASC-cultured gels for more than 2 weeks. Even though the GNTs in ASC/gels (at $750 \mathrm{~nm}$ ) showed significantly brighter PA signals on day 16 , it was shown here that the degradation of PFGs, which occurred in the second week of the culture period, correlated with the increased PA signals due to the increased concentration of GNTs/volume of gel. The total amount of gold, analyzed using inductively coupled plasma mass spectrometry, from individual gels digested at each time point did not change dramatically (data not shown). This result confirms the quantified total PA signal.

The PA imaging technique applied here has several advantages compared to other current imaging modalities. First, the PA imaging methodology is nonionizing and capable of performing imaging with long-term tracking ability, as demonstrated here (16 days). PA imaging can quantify and compare the signal intensity between test groups, as shown in the current study and work by Nam et al on BMSCs. ${ }^{2}$ In addition, PA imaging has greater spatial sensitivity, requiring relatively small numbers of labeled cell samples. Moreover, PA imaging also allows for superior temporal resolution compared to other noninvasive imaging techniques (eg, magnetic resonance imaging).

The current study confirmed work by Jiang et al, who reported that gold nanoparticles are taken up by endocytosis with the resulting structures residing in the cytoplasm. ${ }^{30}$ Compared to the absorbance of GNTs in solution, the absorbance of GNTs in cells showed red shifting and peak broadening (Figure $2 \mathrm{C}$ and $\mathrm{D}$ ). This is due to the aggregation-induced plasmon resonance coupling, a phenomenon which is well described in the literature. ${ }^{31-32}$ In addition, GNTs in the cells showed higher UV-visible absorbance than cells without GNT treatment. The effects of GNTs on cellular behavior and function can be varied depending on the cell type and particle features such as size, shape, and surface characteristics. ${ }^{31}$ Adverse effects such as organelle disruption, membrane breakdown, and apoptosis can be induced. ${ }^{30}$ However, the current study demonstrated no significant differences between ASCs loaded with GNTs compared to those without GNTs in respect to cell morphology, viability, or proliferation. In addition, both the current study and the authors' prior work using BMSCs demonstrate that exocytosis of GNTs does not contribute significantly to a signal loss relative to cellular proliferation. ${ }^{6}$ However, the possibility that aggregated GNTs may be exocytosed, bind to the gel matrix, and contribute to the measured PA signal prior to disaggregation is unable to be ruled out.

The paracrine secretion profile of seeded cells delivered via a tissue-engineered device into damaged tissues can be a critical cue for tissue regeneration. In particular, the therapeutic effects of ASCs on vascular or dermal tissue regeneration have been demonstrated. Several groups reported the enhanced healing effects by treatment with ASC-conditioned media. Park et al analyzed several proteins (cytokines and extracellular matrix proteins) collected from monolayer cultured ASCs $\left(4 \times 10^{5}\right.$ cells on $100 \mathrm{~mm}$ dish $)$ for 72 hours, demonstrating a composition of primarily $809 \mathrm{pg} / \mathrm{mL}$ VEGF, $670 \mathrm{pg} / \mathrm{mL}$ hepatocyte growth factor, $1466 \mathrm{ng} / \mathrm{mL}$ fibronectin, and $921 \mathrm{ng} / \mathrm{mL}$ type I collagen. ${ }^{33}$ They applied US imaging to evaluate dermal tissue but did not label injected materials or cells. According to Nie et al's study related to wound healing, ASCs stimulated blood vessel formation and skin regeneration with an enhanced secretion of soluble factors such as VEGF. ${ }^{16}$ VEGF is the 


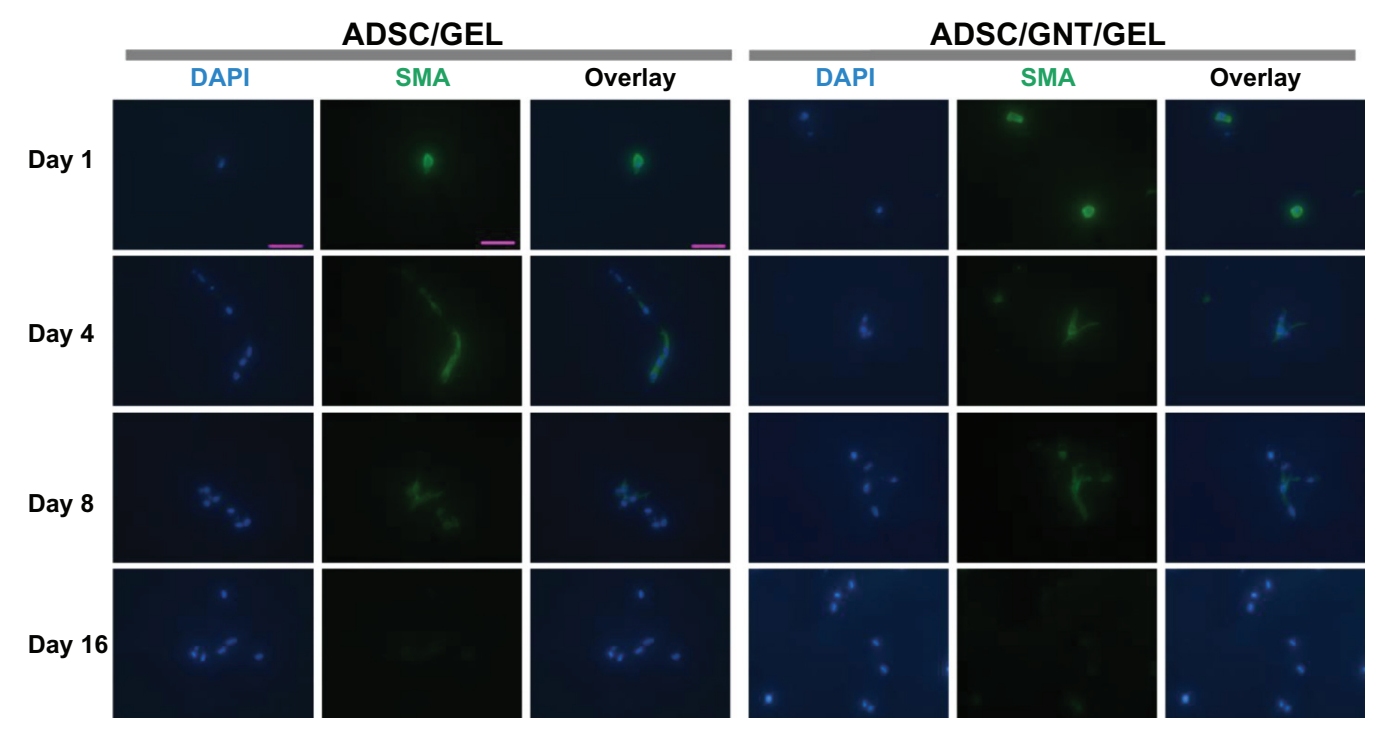

Figure 9 Immunofluorescence staining of smooth muscle $\alpha$-actin over time (days one, four, eight, and I6).

Abbreviations: ADSC, adipose-derived stem cell; DAPI, 4',6-diamidino-2-phenylindole; GNT, gold nanotracer; SMA, smooth muscle $\alpha$-actin.

most critical angiogenic growth factor and plays a role in promoting endothelial cell activity. In the current study, ASCs in the PFG maintained their paracrine effects, which are beneficial for angiogenesis, by showing comparable VEGF secretion levels in a PFG 3D in vitro system at each time point for longer than 2 weeks.

In addition to VEGF, MMP molecules serve as important chemical cues for angiogenesis by promoting matrix degradation. MMPs (eg, MMP-2 and MMP-9) are known to break down extracellular matrix proteins to provide the opportunity for endothelial cell intrusion and blood vessel recruitment. ${ }^{34}$ However, in terms of angiogenesis, the correlation between specific MMPs and VEGF in ASCs has not yet been investigated. The current study demonstrated that ASCs could produce similar secretion profiles of VEGF and MMP-2, even though MMP-2 and MMP-9 showed different trends over time. This secretory capacity of ASCs is likely to serve an important role in wound healing and angiogenesis induction. While it is known that diverse growth factors and enzymes are involved in angiogenesis, the correlation between the release profile of stem cells and the resulting angiogenic process is still not fully understood. The secretory profile evaluated here is not exhaustive, but targets two important classes of molecules and serves to establish the utility of GNT labeling of ASCs.

ASCs are known to express pericyte markers such as SMA which are characteristic of mesenchymal phenotypes. ${ }^{35}$ To evaluate the alteration in SMA expression by GNT treatment into ASCs, SMA immunofluorescence was analyzed over time. GNT loading did not cause modification of SMA expression in ASCs. However, dramatically decreased SMA levels in ASCs either with or without GNT treatment on day 16 were observed. This finding demonstrates that while GNT does not alter the regulation of SMA, a pericytic protein, in $\mathrm{ASCs}$, it is evident that ASCs undergo phenotypic changes in the 3D PFG during cultivation. According to Natesan et al, without any chemical stimulation, cultivation in a PFG induced the upregulation of vascular genes and proteins in ASCs. ${ }^{14}$ The decrease in SMA expression may coincide with morphologic and phenotypic changes characteristic of differentiation towards an endothelial phenotype. However, to clarify the differentiation and paracrine effects of ASCs over time, further studies should focus on investigating the expression of diverse proteins related to angiogenesis and wound healing in ASCs cultured in the PFG with comparison to other matrices such as collagen.

\section{Conclusion}

In conclusion, the current study demonstrated that ASCs in a $3 \mathrm{D}$ PFG platform could be tracked effectively using GNTs and a multimodal biomedical imaging technique without any measurable biological side effects. In the future, gold nanomaterials should be optimized to enhance the cellular uptake efficiency and the quality and sensitivity of imaging for in vivo applications. This report expands the understanding of a next-generation multifunctional strategy, which enables the noninvasive monitoring of stem cell activities and allows for maintenance of their regenerative capacity for tissue engineering applications. 


\section{Acknowledgments}

Funding for this work was provided by the TATRC Foundation (W81XWH-09-2-0103), Deployment Related Medical Research Program (W81XWH-09-1-0607), and National Institutes of Health (1R01EB015007). In addition, the authors appreciate the help of Dr Dwight Romanovicz in the ICMB core facility for the ultrathin sectioning of the transmission electron microscope samples.

\section{Disclosure}

The authors report no conflicts of interest in this work.

\section{References}

1. Espandar L, Bunnell B, Wang GY, Gregory P, McBride C, Moshirfar M. Adipose-derived stem cells on hyaluronic acid-derived scaffold: a new horizon in bioengineered cornea. Arch Ophthalmol. 2012;130(2): 202-208.

2. Nam SY, Ricles LM, Suggs LJ, Emelianov SY. In vivo ultrasound and photoacoustic monitoring of mesenchymal stem cells labeled with gold nanotracers. PLoS One. 2012;7(5):e37267.

3. Lalande C, Miraux S, Derkaoui SM, et al. Magnetic resonance imaging tracking of human adipose derived stromal cells within threedimensional scaffolds for bone tissue engineering. Eur Cell Mater. 2011;21: 341-354.

4. Kim K, Jeong CG, Hollister SJ. Non-invasive monitoring of tissue scaffold degradation using ultrasound elasticity imaging. Acta Biomater. 2008;4(4):783-790.

5. Kreitz S, Dohmen G, Hasken S, Schmitz-Rode T, Mela P, Jockenhoevel S. Nondestructive method to evaluate the collagen content of fibrin-based tissue engineered structures via ultrasound. Tissue Eng Part C Methods. 2011;17(10):1021-1026.

6. Ricles LM, Nam SY, Sokolov K, Emelianov SY, Suggs LJ. Function of mesenchymal stem cells following loading of gold nanotracers. Int J Nanomedicine. 2011;6:407-416.

7. Lequeux C, Oni G, Mojallal A, Damour O, Brown SA. Adipose derived stem cells: efficiency, toxicity, stability of BrdU labeling and effects on self-renewal and adipose differentiation. Mol Cell Biochem. 2011;351(1-2):65-75.

8. Cai X, Paratala BS, Hu S, Sitharaman B, Wang LV. Multiscale photoacoustic microscopy of single-walled carbon nanotube-incorporated tissue engineering scaffolds. Tissue Eng Part C Methods. 2012;18(4): 310-317.

9. Meincke M, Schlorf T, Kossel E, Jansen O, Glueer CC, Mentlein R. Iron oxide-loaded liposomes for MR imaging. Front Biosci. 2008;13 4002-4008.

10. Balakumaran A, Pawelczyk E, Ren J, et al. Superparamagnetic iron oxide nanoparticles labeling of bone marrow stromal (mesenchymal) cells does not affect their "stemness." PLoS One. 2010;5(7):e11462.

11. Yukawa H, Mizufune S, Mamori C, et al. Quantum dots for labeling adipose tissue-derived stem cells. Cell Transplant. 2009;18(5): 591-599.

12. Tholpady SS, Katz AJ, Ogle RC. Mesenchymal stem cells from rat visceral fat exhibit multipotential differentiation in vitro. Anat $\operatorname{Rec} A$ Discov Mol Cell Evol Biol. 2003;272(1):398-402.

13. Strem BM, Hicok KC, Zhu M, et al. Multipotential differentiation of adipose tissue-derived stem cells. Keio J Med. 2005;54(3): 132-141.

14. Natesan S, Zhang G, Baer DG, Walters TJ, Christy RJ, Suggs LJ. A bilayer construct controls adipose-derived stem cell differentiation into endothelial cells and pericytes without growth factor stimulation. Tissue Eng Part A. 2011;17(7-8):941-953.
15. Kachgal S, Putnam AJ. Mesenchymal stem cells from adipose and bone marrow promote angiogenesis via distinct cytokine and protease expression mechanisms. Angiogenesis. 2011;14(1):47-59.

16. Nie C, Yang D, Xu J, Si Z, Jin X, Zhang J. Locally administered adiposederived stem cells accelerate wound healing through differentiation and vasculogenesis. Cell Transplant. 2011;20(2):205-216.

17. Fu X, Fang L, Li H, Li X, Cheng B, Sheng Z. Adipose tissue extract enhances skin wound healing. Wound Repair Regen. 2007;15(4):540-548.

18. Fischer LJ, McIlhenny S, Tulenko T, et al. Endothelial differentiation of adipose-derived stem cells: effects of endothelial cell growth supplement and shear force. J Surg Res. 2009;152(1):157-166.

19. Park BS, Jang KA, Sung JH, et al. Adipose-derived stem cells and their secretory factors as a promising therapy for skin aging. Dermatol Surg. 2008;34(10):1323-1326.

20. Lee SH, Jin SY, Song JS, Seo KK, Cho KH. Paracrine effects of adipose-derived stem cells on keratinocytes and dermal fibroblasts. Ann Dermatol. 2012;24(2):136-143.

21. Kachgal S, Carrion B, Janson IA, Putnam AJ. Bone marrow stromal cells stimulate an angiogenic program that requires endothelial MT1MMP. J Cell Physiol. 2012;227(11):3546-3555.

22. Nakatsu MN, Sainson RC, Aoto JN, et al. Angiogenic sprouting and capillary lumen formation modeled by human umbilical vein endothelial cells (HUVEC) in fibrin gels: the role of fibroblasts and angiopoietin-1. Microvasc Res. 2003;66(2):102-112.

23. Jung SN, Rhie JW, Kwon H, et al. In vivo cartilage formation using chondrogenic-differentiated human adipose-derived mesenchymal stem cells mixed with fibrin glue. J Craniofac Surg. 2010;21(2):468-472.

24. Peterbauer-Scherb A, Danzer M, Gabriel C, van Griensven M, Redl H, Wolbank S. In vitro adipogenesis of adipose-derived stem cells in 3D fibrin matrix of low component concentration. J Tissue Eng Regen Med. 2012;6(6):434-442.

25. Currie LJ, Sharpe JR, Martin R. The use of fibrin glue in skin grafts and tissue-engineered skin replacements: a review. Plast Reconstr Surg. 2001;108(6):1713-1726.

26. Zhang G, Drinnan CT, Geuss LR, Suggs LJ. Vascular differentiation of bone marrow stem cells is directed by a tunable three-dimensional matrix. Acta Biomater. 2010;6(9):3395-3403.

27. Zhang G, Wang X, Wang Z, Zhang J, Suggs L. A PEGylated fibrin patch for mesenchymal stem cell delivery. Tissue Eng. 2006;12(1):9-19.

28. Seetharaman S, Natesan S, Stowers RS, et al. A PEGylated fibrinbased wound dressing with antimicrobial and angiogenic activity. Acta Biomater. 2011;7(7):2787-2796.

29. Wolbank S, Peterbauer A, Wassermann E, et al. Labelling of human adipose-derived stem cells for non-invasive in vivo cell tracking. Cell Tissue Bank. 2007;8(3):163-177.

30. Jiang XM, Wang LM, Chen CY. Cellular uptake, intracellular trafficking and biological responses of gold nanoparticles. J Chin Chem Soc. 2011;58(3):273-281.

31. Tam JM, Tam JO, Murthy A, et al. Controlled assembly of biodegradable plasmonic nanoclusters for near-infrared imaging and therapeutic applications. ACS Nano. 2010;4(4):2178-2184.

32. Mallidi S, Larson T, Aaron J, Sokolov K, Emelianov S. Molecular specific optoacoustic imaging with plasmonic nanoparticles. Opt Express. 2007;15(11):6583-6588.

33. Park BS, Jang KA, Sung JH, et al. Adipose-derived stem cells and their secretory factors as a promising therapy for skin aging. Dermatol Surg. 2008;34(10):1323-1326.

34. Dao Thi MU, Trocme C, Montmasson MP, Fanchon E, Toussaint B, Tracqui P. Investigating metalloproteinases MMP-2 and MMP-9 mechanosensitivity to feedback loops involved in the regulation of in vitro angiogenesis by endogenous mechanical stresses. Acta Biotheor. 2012;60(1-2):21-40.

35. Traktuev DO, Merfeld-Clauss S, Li J, et al. A population of multipotent CD34-positive adipose stromal cells share pericyte and mesenchymal surface markers, reside in a periendothelial location, and stabilize endothelial networks. Circ Res. 2008;102(1):77-85. 


\section{Publish your work in this journal}

The International Journal of Nanomedicine is an international, peerreviewed journal focusing on the application of nanotechnology in diagnostics, therapeutics, and drug delivery systems throughout the biomedical field. This journal is indexed on PubMed Central, MedLine, CAS, SciSearch $\AA$, Current Contents ${ }^{\circledR} /$ Clinical Medicine,

Journal Citation Reports/Science Edition, EMBase, Scopus and the Elsevier Bibliographic databases. The manuscript management system is completely online and includes a very quick and fair peer-review system, which is all easy to use. Visit http://www.dovepress.com/ testimonials.php to read real quotes from published authors.

Submit your manuscript here: http://www.dovepress.com/international-journal-of-nanomedicine-journal 